\title{
Review of 23 patients affected by the stiff man syndrome: clinical subdivision into stiff trunk (man) syndrome, stiff limb syndrome, and progressive encephalomyelitis with rigidity
}

\author{
R A Barker, T Revesz, M Thom, C D Marsden, P Brown
}

\begin{abstract}
Objective-To investigate whether the stiff limb syndrome may be separated from the stiff man syndrome and progressive encephalomyelitis with rigidity on simple clinical grounds, and whether such a distinction has implications for aetiology, treatment, and prognosis.
\end{abstract}

Methods - Twenty three patients referred over a 10 year period with rigidity and spasms in association with continuous motor unit activity, but without evidence of neuromyotonia, extrapyramidal or pyramidal dysfunction or focal lesions of the spinal cord were reviewed. The patients were divided into those with an acute or subacute illness, leading to death within 1 year, and those with a chronic course. The latter were divided into those in whom rigidity and spasms dominated in the axial muscles, or in one or more distal limbs, at the time of their first assessment. Results-This simple division identified three distinct groups of patients. (1) Progressive encephalomyelitis with rigidity: two patients had a rapidly progressive condition characterised by widespread rigidity which resulted in death within 6 and 16 weeks. One patient had negative anti-GAD and anti-neuronal antibodies, but had markedly abnormal CSF and widespread denervation. The principal pathological findings in this case were a subacute encephalomyelitis which primarily affected the grey matter. In the remaining patient anti-GAD antibodies were not tested, and postmortem was refused. (2) Stiff man syndrome: eight patients had rigidity and painful spasms of the lumbar paraspinal, abdominal, and occasionally proximal leg muscles associated with a lumbar hyperlordosis. There was no involvement of the upper limbs, distal lower limbs, sphincters or cranial nerves. Seven had anti-GAD antibodies and most had additional evidence of autoimmune disease. Neurophysiologically there was continuous motor unit activity with abnormal exteroceptive reflexes, but a normal interference pattern during spasms. The patients all responded to baclofen/diazepam and remained ambulant. (3) Stiff limb syndrome: thirteen patients had rigidity, painful spasm, and abnormal postures of the distal limb, usually the leg. About half went on to develop sphincter or brainstem involvement. Generalised myoclonic jerks were not a feature. Only two had truncal rigidity, and another two had anti-GAD antibodies. Most had no evidence of autoimmune disease. Neurophysiologically they had continuous motor unit activity in the affected limb, abnormal exteroceptive reflexes, and abnormally segmented EMG activity during spasms. The disease ran a protracted course, and most patients had only a partial response to baclofen or diazepam. About half became wheelchair bound.

Conclusions-The stiff limb syndrome seems distinct from the stiff man syndrome or progressive encephalomyelitis with rigidity, and is an important cause of rigidity and spasm in the setting of continuous motor unit activity.

(F Neurol Neurosurg Psychiatry 1998;65:633-640)

Keywords: stiff man syndrome; progressive encephalomyelitis with rigidity; stiff limb syndrome

The combination of rigidity, reflex, and action induced spasms and continuous motor unit activity at rest is rare, and may be due to tetanus, encephalomyelitis, corticobasal degeneration, focal lesions of the spinal cord, or the stiff man syndrome. Stiff man syndrome is a chronic disorder involving stiffness and painful spasms of the trunk. ${ }^{1}$ An exaggerated lumbar lordosis is an almost universal finding, but signs of cerebral, brainstem, or long tract disturbance are absent. The diagnosis is supported by the finding of anti-GAD antibodies in blood and CSF. However, most series to date report the presence of anti-GAD antibodies in only around $60 \%$ of patients. ${ }^{23}$ Moreover, many patients do not show the classic axial distribution of stiffness and spasms. Recently, Brown et al reported a small series of patients in whom stiffness and spasms remained confined to the lower limbs. ${ }^{4}$ These patients were anti-GAD negative, and it was suggested that they represented a distinct syndrome, possibly due to a chronic spinal interneuronitis. Here, we review the 23 patients of stiff people who presented to our hospital over a 10 year period, in an attempt to redefine the conditions that may present with rigidity and spasm in association with continuous motor unit activity at rest. 
Table 1 Clinical features in stiff people

\begin{tabular}{|c|c|c|c|}
\hline & $\begin{array}{l}\text { Chronic axial } \\
\text { stiffness and } \\
\text { spasm (stiff } \\
\text { man syndrome) }\end{array}$ & $\begin{array}{l}\text { Chronic stiffness } \\
\text { and spasms of } \\
\text { distal limb (stiff } \\
\text { limb syndrome) }\end{array}$ & $\begin{array}{l}\text { Progressive } \\
\text { encephalomyelitis } \\
\text { with rigidity }\end{array}$ \\
\hline Number & 8 & 13 & 2 \\
\hline $\operatorname{Sex}(M: F)$ & $5: 3$ & $5: 8$ & $2: 0$ \\
\hline Age (y, mean (range)) & $36(22-47)$ & $41(18-70)$ & 57 \\
\hline Duration (y, mean (range)) & $7.5(1-14)$ & $6.2(1-19)$ & 0.2 \\
\hline \multicolumn{4}{|l|}{ Site of first symptom (n (\%)): } \\
\hline Bulbar & 0 & 0 & $2 \ddagger$ \\
\hline Back & $7(88)$ & $1(8)$ & \\
\hline Limb & $1(12)$ & $12(92)$ & \\
\hline Preceding illness (n (\%)) & 0 & $5(39)$ & 0 \\
\hline Relapsing remitting course (n (\%)) & $1(12)$ & $7(54)$ & 0 \\
\hline Brainstem involvement $(\mathrm{n}(\%))^{\star}$ & 0 & $5(39)$ & 2 \\
\hline Upper limb involvement $(\mathrm{n}(\%))^{\star}$ & 0 & $10(77)$ & 2 \\
\hline Sphincter involvement $(\mathrm{n}(\%))^{\star}$ & 0 & $7(54)$ & $0 / 1 \rrbracket$ \\
\hline Lumbar hyperlordosis (n (\%)) & $7(88)$ & $2(15)$ & 0 \\
\hline Truncal rigidity $(\mathrm{n}(\%))$ & $8(100)$ & $2(15)$ & 2 \\
\hline Fixed posture of distal limb (n (\%)) & 0 & $13(100)$ & 2 \\
\hline Number wheelchair bound (n (\%)) & $0 \dagger$ & $6(46)$ & $1 \Phi$ \\
\hline Number died & 0 & 0 & 2 \\
\hline
\end{tabular}

${ }^{\star}$ Either historically or on examination.

†One patient with stiff man syndrome required a wheelchair for outdoor use.

$\ddagger$ Both these patients had bulbar symptoms initially but they were rapidly followed by limb involvement that dominated the clinical presentation of these patients.

\$The index case deteriorated so rapidly that no assessment of sphincter function was possible and the need for a wheelchair was not applicable.

Unless otherwise specified, the figures in parentheses refer to the number of patients affected out of the total with each condition.

\section{Patients and methods}

All patients referred to this hospital between 1986 and 1995 with a diagnosis of possible stiff man syndrome were analysed. Patients were examined by the authors and those with extrapyramidal disease, spasticity, corticobasal degeneration, dystonia, reflex sympathetic dystrophy, neuromyotonia, or structural lesions of the spinal cord were excluded. The 23 patients identified all had, at some point, evidence of continuous motor unit activity and were analysed in terms of their clinical features, investigations, response to therapy, and natural history. Six patients were no longer under follow up at this hospital, and in these patients their current clinical condition was assessed through contact with the patients' general practitioner or local neurologist. Two patients died. Four of the patients with distal stiffness, and two of those with axial stiffness have been previously described. ${ }^{4-6}$

Anti-GAD antibodies were measured by immunoprecipitation of a radiolabelled recombinant human GAD 65 antigen. Details of the techniques used for surface polymyography, and the recording of cutaneomuscular and startle responses have been previously published. ${ }^{4}$ The response to therapy was assessed using the medical notes, as no standard objective measure of response was employed in all patients. Such a response was graded in terms of either (a) no response; (b) improvement in stiffness and spasms with only a transient improvement in mobility; and (c) a sustained improvement in mobility. Limited sample sizes precluded useful statistical analysis.

\section{Results}

A total of 23 patients were entered into the study for analysis. After review, it became apparent that these patients could be divided into two groups: those with progressive encephalomyelitis with rigidity, an acute or suba- cute illness leading to death within 1 year, and those with a chronic course. The second group were further divided into those in whom rigidity and spasms dominated in the axial muscles (of the neck, trunk, and proximal lower limb), and those in whom rigidity and spasms dominated in one or more distal limbs, by the time of their first assessment ( 3 months to 4 years after onset of the illness). In practice the distinction was relatively absolute, with only two out of the 13 patients with stiff limbs having any evidence of axial rigidity. In one of these patients axial difficulties had been delayed by several years and, in both they were overshadowed by distal stiffness and spasm by the time of their first assessment. Patients with axial rigidity met the criteria of Lorish et al for the diagnosis of the stiff man syndrome, with the exception of treatment response, which we do not consider an absolute prerequisite for the diagnosis. ${ }^{7}$ Those with distal rigidity had a condition similar to that described by Brown et $a l .{ }^{4}$ We call this the stiff limb syndrome.

\section{CLINICAL FEATURES}

There were no distinct differences in the sex distribution or age of patients with either axial or distal rigidity (table 1). The duration of illness (1 to 19 years) was also little different between these two groups, although the patients with progressive encephalomyelitis with rigidity succumbed rapidly to his disorder, both dying within weeks of developing their first symptoms of rigidity.

The presenting features of these different disorders were almost entirely in accord with the differential distribution of rigidity on examination. Thus seven out of eight patients with axial stiffness and the stiff man syndrome complained of stiffness and painful spasms of their lower back with some involvement of their proximal legs, and associated difficulty in walking. The initial symptom in one patient in this group was stiffness of the whole leg, but marked involvement of the lumbar paraspinal and abdominal musculature dominated the clinical picture by the time of his first assessment. By contrast, the initial complaint in patients with distal rigidity (stiff limb syndrome) was stiffness and painful spasm of a limb, usually of the calf and foot, with difficulty walking. Spasms in all groups could be spontaneous, reflex, or provoked by voluntary action. Although spasms could have a jerky quality, particularly in those with distal rigidity, none of the patients had generalised myoclonic jerks.

All but one patient with axial rigidity had an exaggerated lumbar lordosis, but none had any fixed abnormal posturing of a distal limb. By contrast, the latter was a universal finding among those with distal rigidity. In two patients the distal arm rather than the leg was involved in this group. In the one patient with distal rigidity but early symptomatic involvement of the truncal musculature, this was not associated with a lumbar hyperlordosis until 3-4 years after the lower limb had become clinically involved. In the other case of involvement of the truncal musculature with distal rigidity, these symptoms developed 16 years after the 
Table 2 Investigative findings in stiff people

\begin{tabular}{llll}
\hline & $\begin{array}{l}\text { Chronic axial } \\
\text { stiffness and } \\
\text { spasm(stiff man } \\
\text { syndrome) } \\
(\%))\end{array}$ & $\begin{array}{l}\text { Chronic stiffness and } \\
\text { spasm of distal limb } \\
\text { (stiff limb syndrome) } \\
(n \text { (\%)) }\end{array}$ & $\begin{array}{c}\text { Progressive } \\
\text { encephalomyelitis with } \\
\text { rigidity (n (\%)) }\end{array}$ \\
\hline Number & 8 & 13 & 2 \\
Diabetes mellitus & $3 / 8(37.5)$ & $0 / 13(0)$ & $0 / 2$ \\
Presence of anti-GAD antibodies & $7 / 8(88)$ & $2 / 13(15)$ & $0 / 1$ \\
Other positive auto-antibody serology* & $6 / 7(86)$ & $5 / 13(39)$ & $1 / 2$ \\
CSF protein $>0.6 \mathrm{~g} / 1$ & $1 / 8(12.5)$ & $4 / 11(36)$ & $1 / 2$ \\
CSF cell count $>5 \mathrm{~cm}^{3}$ & $1 / 8(12.5)$ & $1 / 11(9)$ & $1 / 2$ \\
CSF oligoclonal bands & $4 / 7(57)$ & $2 / 12(17)$ & $1 / 2$ \\
MRI brain abnormality & $2 / 5(40)$ & $1 / 12(9)$ & $1 / 2$ \\
MRI spine abnormality & $0 / 5(0)$ & $2 / 12(18)$ & ND \\
Denervation on EMG & $0 / 5(0)$ & $2 / 12(17)$ & ND \\
Abnormal CMCT & $0 / 6(0)$ & $1 / 8(13)$ & ND \\
SEP (lower limb) abnormality & $0 / 4(0)$ & $1 / 8(13)$ & ND \\
Continuous motor unit activity: & $8 / 8(100)$ & $4(31)$ & ND \\
$\quad$ Back and abdomen & $4 / 8(50)$ & $13(100)$ & ND \\
Proximal or distal limb & $8 / 8(100)$ & $10 / 12(83)$ & $7 / 11(63)$ \\
Abnormal cutaneomuscular reflexes from the lower limbs & $4 / 6(67)$ & $9 / 12(75)$ & \\
Abnormal startle response & $0 / 6(0)$ & & \\
Abnormal interference pattern during spasms & & & \\
\hline
\end{tabular}

${ }^{\star}$ For details of tests for autoantibodies see text.

$\mathrm{ND}=$ not done; $\mathrm{n}=$ the number of patients affected out of those tested.

onset of the disease. Progressive encephalomyelitis with rigidity began with bulbar involvement. Within days to weeks the limbs, then the truncal muscles were affected.

The natural history was different in the three groups. Patients with axial stiffness (stiff man syndrome) seemed to progress and then stabilise after months to years. By contrast, seven patients with distal stiffness (stiff limb syndrome) followed a more relapsing and remitting course. Most patients had isolated stiffness and spasms of the lower limbs for several years, but, in time, about three quarters of the patients developed involvement of the upper limb, half developed mild sphincter disturbance (urinary frequency, urgency, and occasional urge incontinence) and almost 40\% developed symptoms or signs of brainstem disturbance (often transient). The mean periods before involvement of the upper limbs, sphincters, or brainstem was 3, 5, and 2 years respectively. Patients with distal rigidity become more disabled over time, with six patients becoming wheelchair bound after a mean of 3.5 years, despite treatment. By contrast none of the patients with chronic axial stiffness (stiff man syndrome) became wheelchair bound. None of the patients with axial or distal stiffness developed any signs of an underlying malignancy.

INVESTIGATIVE FINDINGS

There were no significant differences in the routine haematological and biochemical blood tests, with the exception of the presence of diabetes mellitus in three patients with axial stiffness (stiff man syndrome; table 2). All but one of the patients with axial rigidity had antibodies to glutamic acid decarboxylase (GAD) in the serum, together with a range of other autoantibodies (including anti-islet cell, thyroid microsomal, gastric parietal cell, and smooth muscle antibodies). Most patients with distal stiffness (stiff limb syndrome), on the other hand, were anti-GAD antibody negative. The two patients in whom anti-GAD antibodies were present were otherwise indistinguishable from other patients with distal stiffness.
Patients with distal rigidity also had a much lower incidence of other autoantibodies.

Just over half of the patients with axial rigidity (stiff man syndrome) had oligoclonal bands confined to CSF whereas only $17 \%$ of patients with distal rigidity (stiff limb syndrome) had such bands in their CSF. The index patient with progressive encephalomyelitis with rigidity had an abnormal CSF from the outset of his rigid syndrome, and as the disease progressed so did the abnormalities in the CSF with an increasing lymphocytosis and persistent oligoclonal bands with a raised protein concentration.

No abnormalities were found on MRI in the vast majority of patients. The brain abnormalities in the remaining patients consisted of one or a few non-specific white matter lesions $(n=3)$, mesial temporal sclerosis in a young patient with epilepsy ${ }^{6}$ and global atrophy $(n=1)$. The only abnormal spinal MRI was in a 37 year old woman with distal stiffness, in whom there were scattered white matter lesions within the cervical cord. The nature of these abnormalities was not clear, but her central motor conduction time and limb somatosensory evoked potentials (SEPs) were normal.

Routine peripheral neurophysiology showed no evidence of a neuropathy or neuromuscular problem; specifically, none of the patients had any evidence of neuromyotonia. The index patient with progressive encephalomyelitis with rigidity had profuse denervation in all four limbs. Central motor conduction time, SEPs from the lower limbs, and visually evoked responses were each abnormal in only one patient.

All patients with axial stiffness (stiff man syndrome) had continuous motor unit activity in their lumbar paraspinal muscles, with half having additional continuous motor unit activity in their proximal lower limbs. By contrast only four patients (31\%) with distal stiffness (stiff limb syndrome) had continuous motor unit activity in their paraspinal muscles, whereas continuous motor unit activity was recorded distally in the affected leg or arm in all 
R tibialis anterior

$\mathbf{L}$ tibialis anterior

R quadriceps

L quadriceps

R lumbar paraspinal

L lumbar paraspinal

$\mathrm{R}$ rectus abdominis

$L$ rectus abdominis
A

\begin{tabular}{c}
\hline+20 \\
\hline 201 \\
\hline
\end{tabular}

B

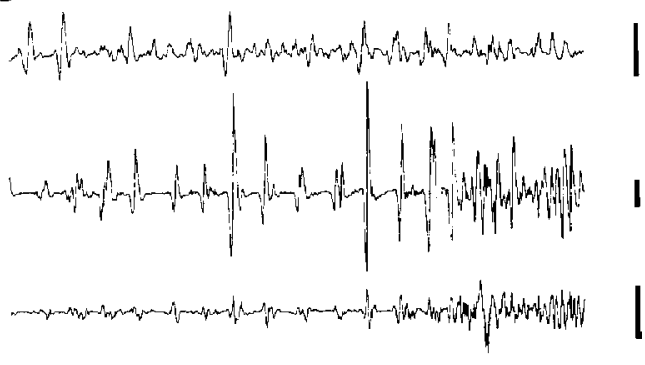

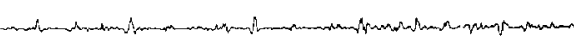

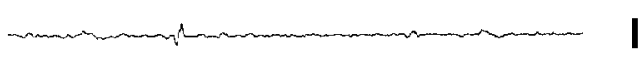

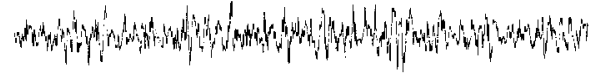

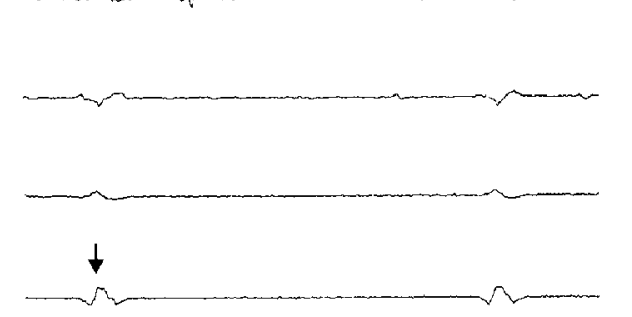

$250 \mathrm{~ms}$

Figure 1 (A) Unrectified surface EMG activity during spontaneous spasms in a patient with chronic axial rigidity (stiff man syndrome) and positive anti-GAD antibodies, and (B) in a patient with rigidity of the distal lower limbs (stiff limb syndrome) who was anti-GAD antibody negative. (A) The spasm is confined to the muscles of the trunk, and EMG activity is indistinguishable from that recorded in a voluntary contraction. (B) The spasm is confined to the lower limbs, and EMG activity in the left $(L)$ tibialis anterior tends to segment into large, but brief discharges. ECG artefact can just be made out in $A$ and is more clearly visible in B (arrowed). Vertical calibrations are $100 \mu \mathrm{V}$ and $500 \mu V$ for the lower and upper four channels respectively.

patients. Continuous motor unit activity tended to be segmented in half of the patients with distal stiffness. The cutaneomuscular reflexes from the tibial nerve at the ankle were virtually always abnormal in both groups with chronic stiffness. Similarly, about two thirds of patients in each group had reflex spasms to unexpected auditory stimuli. However, the nature of the EMG activity recorded during action and reflex induced spasms did serve to distinguish between the two groups (fig 1). In those patients with axial stiffness (stiff man syndrome) the EMG discharge was indistinguishable from a normal interference pattern, but in three quarters of patients with distal stiffness (stiff limb syndrome) the EMG was abnormally segmented, consisting of the grouped discharges of many motor units (often an exacerbation of the resting discharge pattern). Repetitive EMG bursts were irregular in all but three of these patients.

RESPONSE TO THERAPY

The index patient with progressive encephalomyelitis with rigidity failed to show any response to high dose baclofen and diazepam, as well as to high dose intravenous methylprednisolone, intravenous immunoglobulin, and plasma exchange. The other patient required diamorphine for control of his painful spasms.

Rigidity and spasms responded to a combination of baclofen and diazepam in all but one of the patients with axial stiffness (stiff man syndrome), the exception being the patient with negative anti-GAD antibodies. Mobility improved in half the patients, and treatment effects were sustained over time. By contrast, two patients with distal stiffness (stiff limb syndrome) did not respond to either or both drugs, and seven only had a partial response. Mobility only improved in a quarter of patients with distal stiffness.

The role of immunosuppression was less clear. Five patients with distal stiffness (stiff limb syndrome) were treated with either oral or intravenous steroids, and only one reported some slight benefit, with another patient describing a definite deterioration. One of the two patients with axial stiffness treated with steroids had a slight benefit, and the other reported no significant change in his condition. The two patients with axial stiffness (stiff man syndrome) treated with plasma exchange reported no benefit, whereas one patient treated with intravenous immunoglobulin responded dramatically. ${ }^{5}$ These treatments were not tried in those with distal stiffness (stiff limb syndrome).

PATHOLOGICAL FINDINGS IN A PATIENT WITH PROGRESSIVE ENCEPHALOMYELITIS WITH RIGIDITY Only one patient came to postmortem. Both lungs were congested with patchy basal consolidation, confirming bronchopneumonia as the immediate cause of death. Thorough examination did not disclose an occult malignancy. The brain weighed $1490 \mathrm{~g}$ and had no atrophy or structural abnormality. Histologically there was chronic leptomeningitis, especially severe in the leptomeninges overlying the 


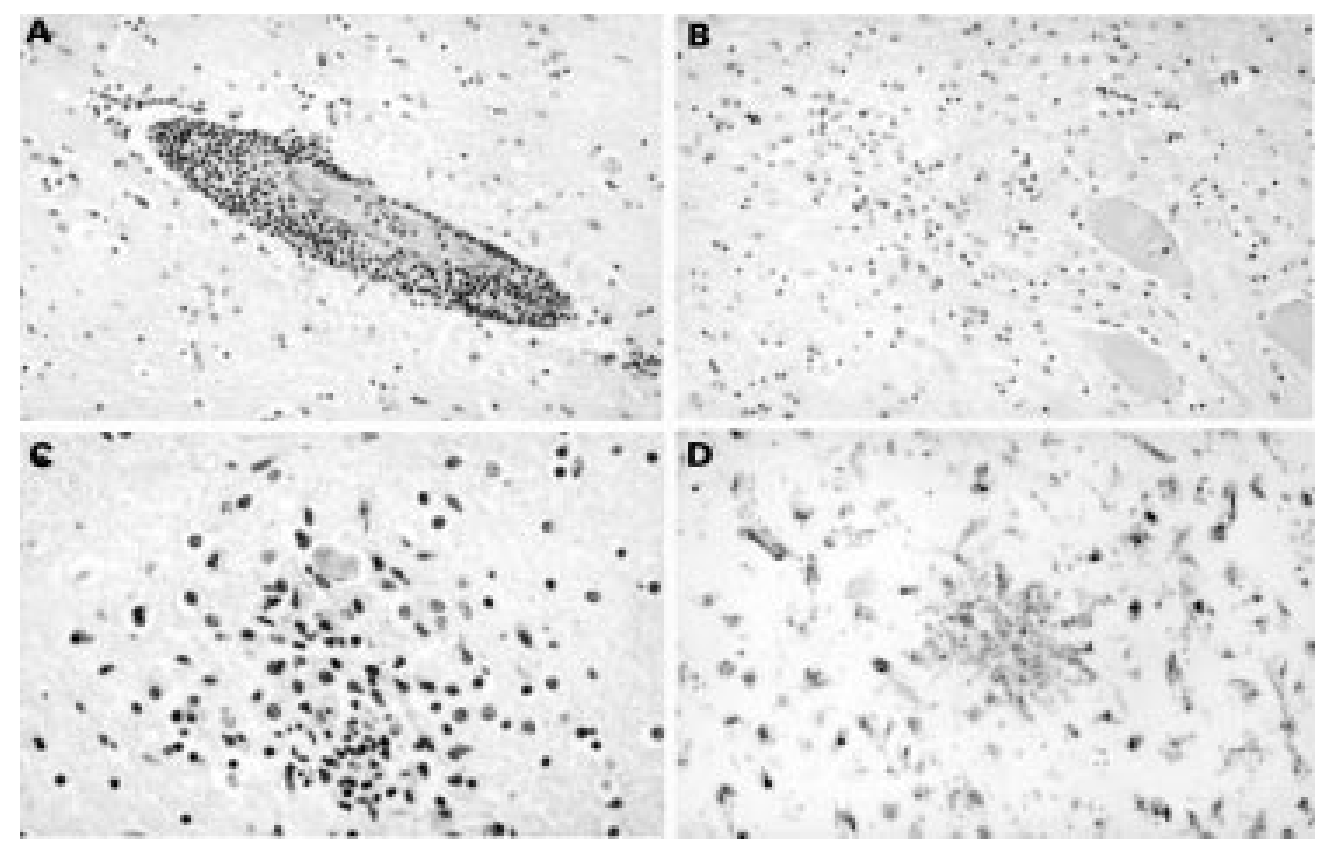

Figure 2 Histology of spinal cord and brain in a patient with progressive encephalomyelitis with rigidity. $(A)$ Blood vessel in the hippocampus surrounded by thick lymphocytic cuff. (B) Microglial proliferation in the cervical cord. (C) Microglial proliferation in the amygdala. Haematoxylin and eosin preparation $(A, B$, and $C)$. (D) Typical microglial module shown by Ham 56 immunohistochemistry. Magnification originally $\times 300$ in $A, B$, and $D$, and $\times 500$ in $C$.

brainstem and spinal cord. There was also evidence for a florid subacute polioencephalitis with frequent cuffs of mature perivascular lymphocytes (mixed T and B cells), plasma cells, and macrophages, as well as neuronophagia, microglial nodules, and diffuse microglial activation accompanied by marked astrogliosis (fig 2). As a consequence there was variable neuronal loss in the affected areas. In the cerebral hemispheres the encephalitic process was most severe in the grey matter of the medial temporal lobe including the hippocampus and amygdala, the anterior cingulum, and insular cortex and similar, although less severe changes, were present in the grey matter of the thalamus, subthalamus, hypothalamus including the mamillary bodies, putamen, globus pallidus, and the nucleus basalis of Meynert. The brainstem showed extensive involvement: the periaqueductal grey matter, the third nerve nucleus, red nucleus, and substantia nigra were affected in the midbrain and the involvement of the periaqueductal grey matter, loci coerulei, nucleus centralis superior, nucleus centralis oralis, processus griseum pontis supralemniscalis, and the griseum pontis was apparent in the pons. The medulla was also among the anatomical areas which were most affected and both microglial nodules and cuffs of perivascular lymphocytes were seen to affect extensively the grey matter underlying the fourth ventricle. The structures involved included the hypoglossal nucleus, the dorsal motor nucleus of the 10th cranial nerve, the medial vestibular nucleus, and the lateral cuneate nucleus. In addition, the medullary reticular formation and inferior olive were also affected. In the lower medulla the hypoglossal nucleus was relatively mildly affected, as were the gracile and cuneate nuclei as well as the nucleus tractus spinalis trigemini. The cerebellum showed moderate Purkinje cell loss with basket cell preservation and gliosis, and microgliosis of the molecular layer. Microglial nodules were noted in the dentate nucleus of the cerebellum.

Several levels representing the cervical, thoracic, lumbar, and sacral cord were examined. All major areas of the spinal cord showed a considerable increase in number of micoglial cells in the grey matter with frequent formation of microglial nodules. The anterior horn cells showed, in general, mild depletion and the intermediolateral cell columns were involved at some levels. The neurons of Clarke's column appeared preserved. Frequent, rather thick perivascular lymphocytic cuffs were present in the lateral and posterior columns at all levels and in the anterior column at some of the levels of the cord examined. The white matter was well myelinated at all levels examined. Special stains for bacteria and fungi were negative as were immunostains for herpes simplex virus, cytomegalovirus, and Toxoplasma gondii.

Autonomic ganglia (superior cervical ganglia, paravertebral sympathetic ganglia and celiac ganglion), showed variable infiltrates of chronic inflammatory cells within the ganglia in mainly perivascular and subcapsular locations. The infiltrates were composed of lymphocytes, plasma cells, and macrophages. This inflammatory process seemed particularly marked in the celiac ganglia where diffuse infiltrates of macrophages were present, surrounding ganglion cells with focal neuronophagia. Dorsal root ganglia at the cervical and thoracic levels disclosed a milder but similar inflammatory picture. It was difficult to assess the extent of nerve cell loss within these peripheral ganglia, but nodules of Nageotte were noted in increased numbers indicating focal depletion. However, the dorsal roots showed no significant depletion of myelinated fibres. Median 
nerve showed focal lymphocytic infiltrates in the endoneurium without any evidence of degeneration.

\section{Discussion}

We were able to identify 23 patients with rigidity and spasms in association with continuous motor unit activity at rest, in whom there was no evidence of neuromyotonia or extrapyramidal or pyramidal dysfunction. Only two of these patients had a subacute course, and postmortem confirmed progressive encephalomyelitis with rigidity in one. The remaining 21 chronic patients were divided into those whose major complaint was axial stiffness and spasm, or stiffness and spasm of a distal limb. It turns out that this simple division serves to identify two groups that can be distinguished in terms of clinical features, investigative findings, and response to therapy. Those with axial stiffness and spasms fulfilled the general criteria of Lorish et al for the diagnosis of the classic stiff man syndrome. ${ }^{7}$ The remainder had a condition akin to the stiff leg syndrome reported by Brown et $a l,{ }^{4}$ although here we show that this condition may also involve the upper limb and brainstem in some patients. Accordingly, we rename this the stiff limb syndrome.

PROGRESSIVE ENCEPHALOMYELITIS WITH RIGIDITY Progressive encephalomyelitis with rigidity is a rare condition characterised by progressive rigidity of both limb and truncal muscles with brainstem involvement, myoclonus, and spasms. ${ }^{8-12}$ Most patients have died within 2-3 years. Both our patients developed bulbar difficulties before the rapid and disseminated involvement of all four limbs and the trunk. One patient came to postmortem. The principal neuropathological findings were those of a subacute encephalomyelitis which primarily, but not exclusively, affected grey matter (polioencephalitis). Of the hemispheric structures the limbic areas were the most affected and the pathological process was also severe in the lower brainstem and spinal cord. In addition, there was ganglionitis affecting both the sympathetic and dorsal root ganglia. Although the anatomical distribution of the histological changes was consistent with a paraneoplastic encephalomyelitis, ${ }^{13}$ the presence of a primary malignancy could not be confirmed at postmortem-a situation not uncommonly found in more well recognised paraneoplastic syndromes. ${ }^{14}$

In our patient the histological changes were, in general, more widespread than in the other pathologically documented patients previously reported with progressive encephalomyelitis with rigidity. ${ }^{8-12}$ However, it is a common finding in all patients including ours that the lower brainstem and spinal cord is severely affected. Although the involvement of the anterior horn cells is documented in some of the patients, the depletion of the spinal inhibitory interneurons, specifically examined in one previous study, may be essential in the pathogenesis. ${ }^{12}$
CHRONIC AXIAL STIFFNESS AND SPASMS

These patients all had the classic stiff man syndrome as first described by Moersch and Woltman. ${ }^{15}$ This presents with paraspinal and abdominal rigidity with a lumbar hyperlordosis and superimposed spasms precipitated by sudden movement, noise, tactile stimulation, or emotional upset. It is associated with normal muscle strength and a normal sensory examination, but continuous motor unit activity is found in the paraspinal muscles. The proximal legs can be involved in the process, and this is often only apparent on walking when the patient has a stiff gait. The calf and foot muscles are rarely, if ever involved. ${ }^{76}$ There is no upper limb, cranial nerve, or sphincter disturbance.

All eight of our patients showed these classic features of stiff man syndrome, with the appropriate neurophysiological abnormalities of continuous motor unit activity and abnormal exteroceptive reflexes. All but one of our patients had anti-GAD antibodies, a figure that is higher than that seen in other studies, ${ }^{23}$ perhaps because of our insistence that rigidity and spasms should predominantly involve the trunk. The association with insulin dependent diabetes mellitus and other autoantibodies was confirmed, with $37.5 \%$ of the patients having insulin dependent diabetes mellitus and $87.5 \%$ having other autoantibodies including anti-islet cell antibodies. ${ }^{17}{ }^{18}$ Most patients responded to a combination therapy of diazepam and baclofen, but the response to immunotherapy was disappointing, except in one patient. Our patients with stiff man syndrome had a typical natural history of slow progression without obvious spontaneous relapses or remissions. None became wheelchair dependent, in agreement with earlier studies. ${ }^{19}$

The pathophysiology of stiff man syndrome is unclear. No histopathological abnormalities have been identified in typical patients. ${ }^{20}$ An autoimmune basis is thought likely in view of the autoantibody profile of many of the patients. $^{2}$ However, it is unclear whether anti-GAD antibodies are central to the pathogenesis of this condition or an epiphenomenon.

CHRONIC STIFFNESS AND SPASMS OF THE DISTAL LIMB

Thirteen patients had stiffness and spasms dominating in one or more distal limbs, as part of an illness lasting up to 19 years. Truncal rigidity and/or hyperlordosis of the lumbar spine were only present in two of these patients, and in these instances were overshadowed by rigidity elsewhere.

Four of the patients have been previously reported in detail by Brown et al, who described a chronic progressive disorder beginning in middle age and involving stiffness and painful spasms of the lower limbs. ${ }^{4}$ Symptoms and signs of brainstem, pyramidal, and sensory dysfunction were absent, and sphincter disturbance developed after many years in only one patient. Likewise, there was little or no electrophysiological evidence of long tract disturbance. However, continuous motor unit activity was present at rest in at least one limb muscle, 
cutaneomuscular reflexes were abnormal, and spasms had a characteristic segmented appearance on EMG. Brown et al suggested that the disorder was due to a chronic spinal interneuronitis. Here we extend the range of this disease, which may begin in the upper limb or lead to late brainstem dysfunction in the minority of patients.

Patients typically presented between the ages of 20 and 50 with a rigid distal limb, usually the lower leg. About half of the patients followed a relapsing and remitting course, and around a third developed symptoms or signs of brainstem involvement. These were usually delayed in onset and transient. Similarly, half developed a sphincter disturbance after a mean of 5 years. None of the patients developed the generalised myoclonus characteristic of the jerking stiff man syndrome. ${ }^{21}$ Cortical and cognitive deficits were absent, as were seizures, with one exception. Half the patients become wheelchair or bed bound after a few years, in marked contrast with those with stiff man syndrome.

The autoimmune profile of patients with stiff limb syndrome also differed from those with stiff man syndrome. There were no patients with insulin dependent diabetes mellitus, and serum autoantibodies were present in only around a third of patients. In addition, only $15 \%$ had anti-GAD antibodies, as opposed to $88 \%$ of our patients with stiff man syndrome. Patients with stiff limbs also had a lower incidence of intrathecal synthesis of immunoglobulin $(17 \% v 57 \%$ of patients with stiff man syndrome), although the total CSF protein was more often raised.

Detailed neurophysiological studies in these patients support the contention that patients with stiff limbs are different from those with stiff man syndrome. Although both groups had continuous motor unit activity at rest, the distribution was very different. In addition, three quarters of patients with stiff limbs had spasms with a characteristic interference pattern, consisting of hypersynchronous segmented discharges. This was not seen in the stiff man syndrome.

These patients therefore have a distinctive clinical and electrophysiological picture. Despite the severity of the limb stiffness and spasms there was a striking scarcity of specific symptoms, signs, or electrophysiological abnormalities attributable to the long tracts of the spinal cord. This has led to the suggestion that the rigidity and the spasms evoked by peripheral somaesthetic stimuli, voluntary action, or startle are due to a localised spinal interneuronitis. ${ }^{4}$ To date, histology has been reported in only one patient with a clinical history consistent with the stiff limb syndrome, and showed an encephalomyelitis predominantly affecting the lumbar cord..$^{22}$ Indeed patients with the idiopathic stiff limb syndrome are very similar to other patients with known focal pathology preferentially involving the grey matter of the spinal cord. Thus intrinsic tumours, ${ }^{23}$ syringomyelia, ${ }^{24}$ vascular insufficiency, ${ }^{25}$ and paraneoplastic myelitis ${ }^{26}$ may be associated with continuous motor unit activity, and reflex and action induced spasms of the trunk and lower limbs. The relatively selective destruction of spinal interneurons in some of these patients has been confirmed histologically. ${ }^{12}{ }^{23}$ Effective stimuli need not be restricted to somaesthetic stimulation below the level of the spinal cord pathology. Paradoxically, jerks and spasms of the legs can be precipitated in patients with spinal lesions by startle inducing stimuli, including sounds. ${ }^{25}$ Presumably this represents an excessive response at the segmental level to descending reticulospinal activity.

Although, with one exception, ${ }^{22}$ pathological reports are lacking in the stiff limb syndrome, the condition has many clinical similarities with the jerking stiff man syndrome, in which there is increasing evidence for a polioencephalomyelitis largely indistinguishable from that found in progressive encephalomyelitis with rigidity. ${ }^{328}$ Patients with the jerking stiff man syndrome can survive more than 10 years, ${ }^{21}$ and may have evidence of an autoimmune diathesis. ${ }^{29}$ Rigidity and spasms often start in and sometimes remain confined to the limbs in this condition. ${ }^{28}$ However, unlike the stiff limb syndrome, the clinical picture in these patients is dominated by marked cranial nerve signs and a characteristic brainstem myoclonus that involves all four limbs. ${ }^{21}$ Perhaps, the essential difference between these patients and the stiff limb syndrome lies in the distribution rather than the nature of the pathology.

Occasionally, rigidity of the limbs (sometimes with later spread to the trunk) is seen in the setting of breast or small cell lung carcinoma. Such patients have antibodies against the vesicle associated protein amphiphysin. ${ }^{30-32}$ Many of the patients in our series presented before the identification of this antibody, ${ }^{30}$ and none of our patients were tested for it. Nethertheless, the long duration of the stiff limb syndrome in our series would be very much against a paraneoplastic aetiology.

\section{Conclusion}

Of our 23 patients about a third had stiff man syndrome, and two had an aggressive progressive encephalomyelitis with rigidity. However, most had a distinct clinical entity that we term the stiff limb syndrome. Such patients present with rigidity and painful spasms of a limb, usually the distal leg, and abnormal fixed posturing of the foot or hand. There is sparing of the truncal musculature, at least in the early stages of the disease, which may follow a relapsing and remitting course. There may be involvement of the brainstem and sphincters, but this is usually delayed by several years. Neurophysiologically, such patients have continuous motor unit activity in the affected limb with abnormal exteroceptive reflexes, and a characteristic interference pattern during spasms. Most patients have neither anti-GAD nor other autoantibodies. The response to diazepam and baclofen is only partial, with half of the patients becoming wheelchair bound. The role of immunosuppressive therapy is currently unknown. The disease is indolent, lasting many years, and may be due to a spinal interneuronitis, although this remains speculative. 
We thank the following for referring some of the patients: Dr L Findley, Dr RS Kocen, Professor CJ Mathias, Professor WI McDonald, Dr JA Morgan-Hughes, Dr P Rudge, Professor M Ron, and Dr J Duncan. We also thank Dr J Land and Dr M Hawa for performing the serological testing, and Dr PD
Thompson and Dr JC Rothwell for their help with some of the electrophysiological recordings.

1 Toro C, Jacobowitz DM, Hallett M. Stiff-man syndrome. Semin Neurol 1994;14:54-8.

2 Solimena M, Folli F, Aparisi R, et al. Autoantibodies to GABA-ergic neurons and pancreatic beta cells in stiff-man syndrome. N Engl f Med 1990;322:1555-60.

3 Meinck HM, Ricker K, Hülser PJ, et al. Stiff man syndrome: clinical and laboratory findings in eight patients. $\mathcal{F}$ Neurol 1994;24:57-66.

4 Brown P, Rothwell JC, Marsden CD. The stiff-leg syndrome. F Neurol Neurosurg Psychiatry 1997;62:31-7.

5 Barker RA, Marsden CD. Successful treatment of stiff man syndrome with intravenous immunoglobulin. 7 Neurol Neurosurg Psychiatry 1997;62:426.

6 Prevett MC, Brown P, Duncan JS. Improvement of stiff-man syndrome with vigabatrin. Neurology 1997;48: stiff-man

7 Lorish TR, Thorsteinsson G, Howard FM. Stiff-man syndrome updated. Mayo Clin Proc 1989;64:629-36.

8 Campbell AMG, Garland H. Subacute myoclonic spinal interneuronitis. If Neurol Neurosurg Psychiatry 1956;19: interneu

9 Kasperek S, Zebrowski S. Stiff-man syndrome and encephalomyelitis: report of a case. Arch Neurol 1971;24: 22-31

10 Lhermitte F, Chain F, Escourolle R, et al. Un nouveau cas de contracture tétaniforme distinct du "stiff man syndrome": etude pharmacologique et neuropathologique d'un cas d'encéphalomyelite à prédominance médullaire. Rev Neurol (Paris) 1973;128:3-21.

11 Whiteley AM, Swash M, Urich H. Progressive encephalomyelitis with rigidity: its relation to subacute myoclonic spinal interneuronitis and the stiff man syndrome. Brain 1976;99:27-42.

12 Howell DA, Lees AJ, Toghill PJ. Spinal internuncial neurones in progressive encephalomyelitis with rigidity. $\mathcal{F}$ neurones in progressive encephalomyelitis with

13 Henson R, Urich H. Cancer and the nervous system. Oxford: Blackwell, 1982.

14 Dalmau J, Graus F, Rosenblum MK, et al. Anti-Huassociated encephalomyelitis/sensory neuronopathy. A clinical study of 71 patients. Medicine 1992;71:59-72.

15 Moersch FP, Woltman HW. Progressive fluctuating muscular rigidity and spasm (stiff-man syndrome): report of case and some observations in 13 other cases. Mayo Clin Proc 1956;31:421-7.
16 Spehlmann R, Norcross K. Stiff-man syndrome. In: Klawans HL, ed. Clinical neuropharmacology. Vol 4 New Klawans HL, ed. Clinical neurophar
York: Raven Press, 1979:109-21.

17 Grimaldi LM, Martino G, Braghi S, et al. Heterogeneity of autoantibodies in stiff man syndrome. Ann Neurol 1993;34: 57-64.

18 Daw K, Ujihara N, Atkinson M, et al. Glutamic acid decarboxylase in stiff-man syndrome and insulin-dependent diabetes mellitus exhibit similarities and differences in epitope recognition. F Immunol 1996;156:818-25.

19 Gordon EE, Januszko DM, Kaufman L. A critical survey of stiff-man syndrome. Am f Med 1967;42:582-99.

20 Trethowan WH, Allsop JL, Turner B. The stiffman syndrome. Arch Neurol 1960;3:448-56.

21 Leigh PN, Rothwell JC, Traub M, et al. A patient with reflex myoclonus and muscle rigidity: jerking stiff-man syndrome. F Neurol Neurosurg Psychiatry 1980;43:1125-31.

22 Armon C, Swanson JW, McLean JM, et al. Subacute encephalomyelitis presenting as stiff-person syndrome: clinical, polygraphic, and pathologic correlations. Mov Disord 1996;11:701-9.

23 Rushworth G, Lishman WA, Trevor Hughes J, et al. Intense rigidity of the arms due to isolation of motorneurones by a spinal tumour. F Neurol Neurosurg Psychiatry 1961;24:13242.

24 Tarlov IM. Rigidity in man due to spinal interneuron loss. Arch Neurol 1967;16:536-43.

25 Davis SM, Murray NMF, Diengoh JV, et al. Stimulussensitive spinal myoclonus. $\mathcal{F}$ Neurol Neurosurg Psychiatry 1987;50:628-31.

26 Roobol TH, Kazzaz BA, Vecht CHJ. Segmental rigidity and spinal myoclonus as a paraneoplastic syndrome. F Neurol Neurosurg Psychiatry 1987;50:628-31.

27 Sikes ZS. Stiff-man syndrome. Diseases of the Nervous System 1959;29:254-8.

28 Fenzi F, Bongiovanni G, Fincati E, et al. Anatomical and clinical study of a case of subacute encephalomyelitis with hyperekplexia syndrome. Ital F Neurol Sci 1988;9:505-8.

29 Burn DJ, Ball J, Lees AJ, et al. A case of progressive encephalomyelitis with rigidity and positive antiglutamic 449-51.

30 Folli F, Solimena M, Cofieli R, et al. Autoantibodies to a $128-\mathrm{kD}$ synaptic protein in three women with the stiff-man syndrome and breast cancer. N Engl f Med 1993;328:54651.

31 Dropcho EJ. Antiamphiphysin antibodies with small-cell lung carcinoma and paraneoplastic encephalomyelitis. Ann Neurol 1996:39:659-67.

32 De Camilli P, Thomas A, Cofiell R. The synaptic vesicle-associated protein amphiphysin is the $128-\mathrm{kD}$ autoantigen of stiff-man syndrome with breast cancer. $\mathcal{F}$ Exp Med 1993;178:2219-23. 